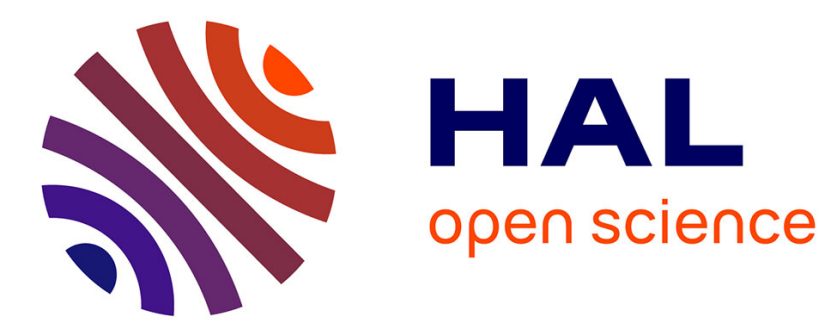

\title{
Creating antibubbles with ultrasound
}

Michiel Postema, Nico de Jong, Georg Schmitz, Annemieke van Wamel

\section{To cite this version:}

Michiel Postema, Nico de Jong, Georg Schmitz, Annemieke van Wamel. Creating antibubbles with ultrasound. IEEE Ultrasonics Symposium, 2005., 2005, Rotterdam, Netherlands. pp.977-980, 10.1109/ULTSYM.2005.1603013 . hal-03193369

\section{HAL Id: hal-03193369 \\ https://hal.science/hal-03193369}

Submitted on 11 Apr 2021

HAL is a multi-disciplinary open access archive for the deposit and dissemination of scientific research documents, whether they are published or not. The documents may come from teaching and research institutions in France or abroad, or from public or private research centers.
L'archive ouverte pluridisciplinaire HAL, est destinée au dépôt et à la diffusion de documents scientifiques de niveau recherche, publiés ou non, émanant des établissements d'enseignement et de recherche français ou étrangers, des laboratoires publics ou privés. 


\title{
Creating antibubbles with ultrasound
}

\author{
Michiel Postema*, Nico de Jong ${ }^{\dagger \ddagger \S}$, Georg Schmitz*, Annemieke van Wamel ${ }^{\dagger \ddagger}$ \\ *Institute for Medical Engineering, Ruhr-Universität Bochum, Bochum, Germany \\ ${ }^{\dagger}$ Department of Experimental Echocardiography, Thoraxcentre, Erasmus MC, Rotterdam, The Netherlands \\ $\ddagger$ Interuniversity Cardiology Institute of the Netherlands, Utrecht, The Netherlands \\ $\S$ Physics of Fluids group, Faculty of Science and Technology, University of Twente, Enschede, The Netherlands
}

\begin{abstract}
Ultrasound contrast agents have been investigated for their potential applications in local drug and gene delivery. A microbubble might act as the vehicle to carry a drug or gene load to a perfused region of interest. The load has to be released with the assistance of ultrasound. We investigate the suitability of antibubbles for ultrasound-assisted local delivery. As opposed to bubbles, antibubbles consist of a liquid core surrounded by a gas encapsulation. Incorporating a liquid drop containing drugs or genes inside an ultrasound contrast agent microbubble, however, is technically challenging.

An ultrasound-insonified microbubble generates a pressure field that is inversely proportional to the distance from the microbubble. Therefore, an oscillating contrast agent microbubble may create a surface instability with a relatively big bubble at a short distance. For big enough instabilities, a drop may be formed inside the big bubble.

Three different contrast agents were subjected to $0.5 \mathrm{MHz}$ ultrasound, with mechanical indices $>0.6$. The contrast agents were inserted through an artificial capillary which led through the acoustic focus of the transducer. High-speed photographs were captured at a speed of 3 million frames per second and higher. We observed that ultrasound contrast microbubbles below resonance size may create visible surface instabilities with bubbles above resonance size. With an albumin-shelled contrast agent, we induced a surface instability that was big enough to create an antibubble inside a free (unencapsulated) gas bubble with an 8 micron diameter. The surface instability has been attributed to the presence of a contrast microbubble with a 3 micron diameter. This instability has the form of a re-entrant jet protruding into the gas bubble. The inward protrusion grew and subsequently drained, leaving a droplet with a five micron diameter inside the bubble. In a subsequent recording after $100 \mathrm{~ms}$, only the gas bubble could be detected. Thus, the lifetime of the antibubble was less then $100 \mathrm{~ms}$. The presence of a surfactant on the interfaces might lead to an improved stability of an antibubble.
\end{abstract}

\section{INTRODUCTION}

Ultrasound contrast agents consist of microscopically small bubbles in the micrometer range. These microbubbles oscillate linearly if insonified at low acoustic amplitudes, but demonstrate highly nonlinear behavior at relatively high acoustic amplitudes, such as rapid collapse, fragmentation and jetting [1]. Therefore, ultrasound contrast agents have been investigated for their potential applications in local drug and gene delivery.

A microbubble might act as the vehicle to carry a drug or gene load to a perfused region of interest. The load has to be released with the assistance of ultrasound.
Several schemes have been proposed to combine microbubbles with a therapeutic load:

\section{A. Mixing microbubbles with a therapeutic agent}

Tachibana et al. subjected cells in the presence of a photosensitive drug to continuous ultrasound, and noticed surface pores on the cells [2]. Inducing porosities in cells with ultrasound, generally referred to as sonoporation, has potential applications in (tumor) cell lysis and selective delivery of drugs and genes into cells.

Independently, it was demonstrated that moderate microbubble oscillations are sufficient to achieve rupture of lipid membranes, in a regimen in which the bubble dynamics can be accurately controlled [3]. This might enable the transport of drugs through the cell membrane.

The behavior of contrast agent microbubbles near cells has been recently studied by Kudo, Van Wamel, and Wolfrum [4], [5], [6].

The in vitro and in vivo transfection of endothelial cells with plasmid DNA mixed with ultrasound contrast agent has been demonstrated [7]. Accelerated cellular uptake of the radionuclide ${ }^{111}$ In-DOTA-Tyr ${ }^{3}$-octreotate takes place when microbubbles are present and under insonification [8].

\section{B. Therapeutic compounds attached to microbubble shells}

It has been put forward that targeted microbubbles with ligands attached to them may be applied for selective delivery to the areas where selective enhancement of the action of ultrasound would be required, and if such a bubble would contain plasmid DNA, enzyme, or another therapeutic agent, it might be released at the site of interest during insonification [9].

A recent advance in local gene delivery is the in vivo delivery of a virus vector, attached to albumin microbubbles [10].

\section{Therapeutic compounds in the gaseous phase}

Sonic cracking is the ultrasound-induced release of gas from thick-shelled microbubbles. Therapeutic compounds in the gas phase might be encapsulated with thick shells, to keep them from dissolving. At the region of interest, the shell can be cracked with ultrasound, releasing the content [11].

A few therapeutic compounds exist in the gaseous phase. We think of nitric oxide [12] and of gaseous anaesthetics. 


\section{Gas-filled lipospheres containing drugs}

May et al. performed high-speed optical experiments on microbubbles with an outer lipid layer, an oil layer, and a gas core [13]. Such microbubbles may be applicable in local drug delivery, since their lipid-oil complex can carry bioactive substances at high concentrations [14]. The delivery of the contents of these microbubbles has been demonstrated in vitro and ex vivo [14].

\section{E. Drug-filled antibubbles}

A therapeutic agent inside the microbubble shell may react with the shell and damp the bubble oscillations. Therefore, it might be more suitable to have the therapeutic agent in the core of the microbubble, separated form the shell by a gaseous layer. Incorporating a liquid drop containing drugs or genes inside an ultrasound contrast agent microbubble, however, is technically challenging. We investigate the suitability of antibubbles for ultrasoud-assisted local delivery.

As opposed to bubbles, antibubbles consist of a liquid core surrounded by a gas encapsulation ( $c f$. Figure 1) [15]. Such a droplet inside a bubble may be generated with the jetting phenomenon: The collapse of a bubble near a free surface produces a liquid jet [16], which may break up into one or several droplets [17]. Early high-speed photographs of jets have been shown by Benjamin and Ellis [18], Crum [19, page 534], and Lauterborn [20], [21]. High-speed photographs of jets breaking up have been presented in [22]. The length of the jet and the bubble pinch-off are related to the jet velocity [23], which in turn is related to the collapsing bubble size [1]. Ultrasound-induced jetting has been observed with ultrasound contrast agent microbubbles with a thin elastic shell [1]. We have also observed jetting with free gas microbubbles that had been released from the thick-shelled ultrasound contrast agent Quantison ${ }^{\mathrm{TM}}$.

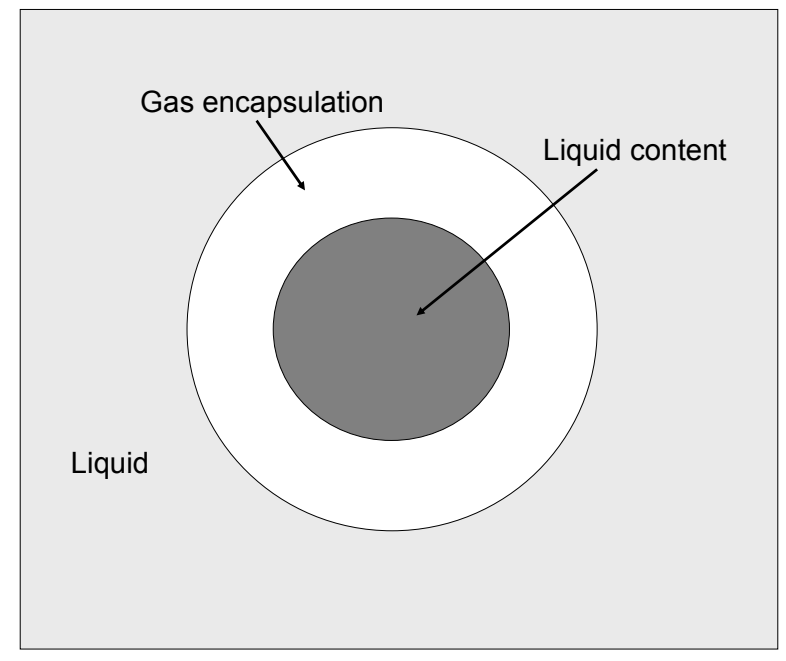

Fig. 1. An antibubble consists of a liquid core encapsulated by a gas shell.

Quantison $^{\mathrm{TM}}$ (Upperton Limited, Nottingham, UK) consists of human serum albumin-encapsulated air bubbles with a mean diameter of $3.2 \mu \mathrm{m}$. Shell thicknesses are between 0.2 and $0.3 \mu \mathrm{m}$ [24]. The pressure field exerted by a Quantison ${ }^{\mathrm{TM}}$ microbubble is very low compared to the ultrasonic field, because of its low oscillation amplitude [11]. A released bubble, however, will generate a very high-pressure, because of its high volumetric acceleration during collapse [25]. Therefore, a collapsing microbubble near a bigger one is expected to generate instabilities on the surface of the bigger one.

\section{EXPERIMENTAL SETUP}

A $5 \mathrm{ml}$ vial containing Quantison ${ }^{\mathrm{TM}}$ (Upperton Limited, Nottingham, UK) was heavily stirred. The agent was inserted through an artificial capillary with a $200 \mu \mathrm{m}$ diameter, without further dilution. The artificial capillary was positioned in a container in the acoustical focus of a V389SU $500 \mathrm{kHz}$ single-element transducer (Panametrics Inc., Waltham, MA), and in the optical focus of a high-numerical aperture microscopic system. An overview hereof is shown in Figure 2.

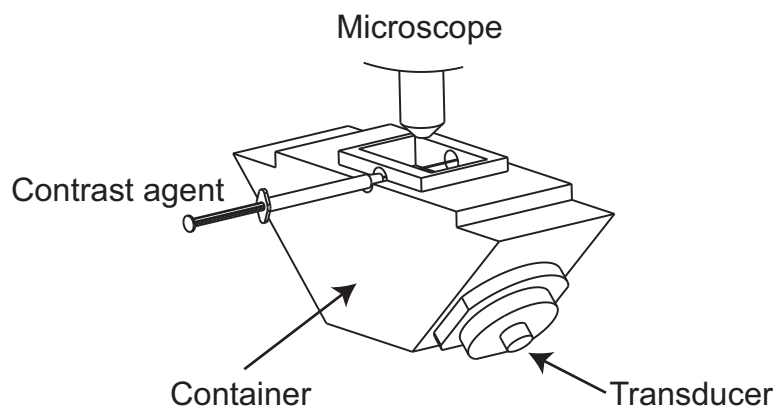

Fig. 2. Overview of the optical observation part of the experimental setup.

Freely flowing microbubbles were insonified with a burst of 8 cycles, $500 \mathrm{kHz}$ ultrasound. Acoustic pressures up to 1.3 $\mathrm{MPa}$ were applied. In this regime, less than $10 \%$ of the Quantison ${ }^{\mathrm{TM}}$ microbubbles demonstrate gas release [11]. Our experimental setup for taking high-speed photographs of microbubbles was more extensively described in [1]. This experiment was performed 13 times. In one experiment, we observed the formation of a drop inside a gas bubble. Here, we present this first result of ultrasonic antibubble generation.

\section{RESULT}

Figure 3 shows a high-speed photographic sequence of a free (unencapsulated) gas microbubble with a diameter of $8 \mu \mathrm{m}$ close to a $3 \mu \mathrm{m}$ Quantison ${ }^{\mathrm{TM}}$ microbubble that is slightly out of optical focus. During insonification with $0.5 \mathrm{MHz}$ ultrasound and a mechanical index $\mathrm{MI}=1.1$, the surface of the free gas microbubble close to the Quantison ${ }^{\mathrm{TM}}$ microbubble becomes instable, followed by the generation of a liquid drop inside the gas microbubble.

In frame 0 , a big free gas microbubble with an $30 \mu \mathrm{m} \mathrm{di-}$ ameter is seen just below a $7.5 \mu \mathrm{m}$ Quantison ${ }^{\mathrm{TM}}$ microbubble that is slightly out of optical focus. After ultrasound arrival, presumably gas release takes place from the Quantison ${ }^{\mathrm{TM}}$ 
microbubble. This new microbubble is seen to expand and contract in the following frames. The two microbubbles interact, leading to an instability at the surface of the big free gas microbubble (frame 2). This instability has the form of a re-entrant jet protruding into the gas microbubble. The inward protrusion grows until frame 4 . Between frames 5 and 8 , the cylindrical protrusion drains. In frame 9, a $5 \mu \mathrm{m}$ droplet is left inside the gas microbubble, while the protrusion retracts. Frame 10 shows the resulting antibubble: a spherical liquid core inside a spherical gas encapsulation.

\section{Discussion}

The jet formation and the bubble pinch-off are similar to the observations of jet break-up in [22]. We attribute the surface instability to the behavior of released gas from the Quantison $^{\mathrm{TM}}$ microbubble. In a subsequent recording after $100 \mathrm{~ms}$, only the gas bubble could be detected. Thus, in this first attempt to generate an antibubble with the aid of an ultrasound contrast agent, the life-time of the antibubble was less then $100 \mathrm{~ms}$. The presence of a surfactant on the interfaces might lead to an improved stability of an antibubble [15]. Techniques for on demand antibubble creation are needed for future applications in therapy.

\section{ACKNOWLEDGMENTS}

The authors are grateful to Upperton Limited, Nottingham, UK, for supplying the contrast agent Quantison ${ }^{\mathrm{TM}}$. This project has been supported by the Technology Foundation STW (RKG.5104).

\section{REFERENCES}

[1] M. Postema, A. van Wamel, C. T. Lancée, and N. de Jong, "Ultrasound-induced encapsulated microbubble phenomena," Ultrasound Med. Biol., vol. 30, no. 6, pp. 827-840, 2004.

[2] K. Tachibana, T. Uchida, K. Ogawa, N. Yamashita, and K. Tamura, "Induction of cell-membrane porosity by ultrasound," Lancet, vol. 353, p. 1409, 1999.

[3] P. Marmottant and S. Hilgenfeldt, "Controlled vesicle deformation and lysis by single oscillating bubbles," Nature, vol. 423, pp. 153-156, 2003.

[4] N. Kudo, T. Miyaoka, K. Okada, K. Yamamoto, and K. Niwa, "Study on mechanism of cell damage caused by microbubbles exposed to ultrasound," Proc. IEEE Ultrason. Symp., pp. 1351-1354, 2002.

[5] A. van Wamel, A. Bouakaz, F. ten Cate, and N. de Jong, "Effects of diagnostic ultrasound parameters on molecular uptake and cell viability," Proc. IEEE Ultrason. Symp., pp. 1387-1390, 2002.

[6] B. Wolfrum, R. Mettin, T. Kurz, and W. Lauterborn, "Observations of pressure-wave-excited contrast agent bubbles in the vicinity of cells," Appl. Phys. Lett., vol. 81, no. 26, pp. 5060-5062, 2002.

[7] Y. Taniyama, K. Tachibana, K. Hiraoka, T. Namba, K. Yamasaki, N. Hashiya, M. Aoki, T. Ogihara, K. Yasufumi, and R. Morishita, "Local delivery of plasmid DNA into rat carotid artery using ultrasound," Circulation, vol. 105, pp. 1233-1239, 2002.

[8] A. van Wamel, A. Bouakaz, B. Bernard, F. ten Cate, and N. de Jong, "Radionuclide tumour therapy with ultrasound contrast microbubbles," Ultrasonics, vol. 42, no. 1-9, pp. 903-906, 2004.

[9] A. L. Klibanov, "Targeted delivery of gas-filled microspheres, contrast agents for ultrasound imaging," Adv. Drug Delivery Rev., vol. 37, pp. 139-157, 1999.

[10] R. V. Shohet, S. Chen, Y. T. Zhou, Z. Wang, R. S. Meidell, R. H. Unger, and P. A. Grayburn, "Echocardiographic destruction of albumin microbubbles directs gene delivery to the myocardium," Circulation, vol. 101, pp. 2554-2556, 2000.
[11] M. Postema, A. Bouakaz, M. Versluis, and N. de Jong, "Ultrasoundinduced gas release from contrast agent microbubbles," IEEE Trans. Ultrason., Ferroelect., Freq. Contr., vol. 52, no. 6, pp. 1035-1041, 2005.

[12] M. Postema, A. Bouakaz, F. ten Cate, G. Schmitz, N. de Jong, and A. van Wamel, "Nitric oxide delivery by ultrasonic cracking: limitations," submitted, 2005.

[13] D. May, J. Allen, and K. Ferrara, "Dynamics and fragmentation of thick-shelled microbubbles," IEEE Trans. Ultrason., Ferroelect., Freq. Contr., vol. 49, no. 10, pp. 1400-1410, 2002.

[14] M. J. Shortencarier, P. A. Dayton, S. H. Bloch, P. A. Schumann, T. O Matsunaga, and K. W. Ferrara, "A method for radiation-force localized drug delivery using gas-filled lipospheres," IEEE Trans. Ultrason., Ferroelect., Freq. Contr., vol. 51, no. 7, pp. 822-831, 2004.

[15] S. Dorbolo, H. Caps, and N. Vandewalle, "Fluid instabilities in the birth and death of antibubbles," New J. Phys., vol. 5, pp. 161.1-161.9, 2003.

[16] J. I. Katz, "Jets from collapsing bubbles," Proc. R. Soc. London A, vol. 455 , pp. 323-328, 1999.

[17] L. Duchemin, S. Popinet, C. Josserand, and S. Zaleski, "Jet formation in bubbles bursting at a free surface," Phys. Fluids, vol. 14, no. 9, pp. 3000-3008, 2002.

[18] T. B. Benjamin and A. T. Ellis, "The collapse of cavitation bubbles and the pressures thereby produced against solid boundaries," Philos. Trans. Roy. Soc. A, vol. 260, pp. 221-240, 1966.

[19] T. G. Leighton, The Acoustic Bubble. London: Academic Press Ltd, 1994.

[20] W. Lauterborn, "Optische Kavitation," Physikalische Blätter, vol. 32, no. 12 , pp. 553-563, 1976.

[21] W. Lauterborn, T. Kurz, R. Mettin, and C. D. Ohl, "Experimental and theoretical bubble dynamics," in Advances in Chemical Physics, I. Prigogine and S. Rice, Eds. Hoboken: John Wiley \& Sons, Inc, 1999, vol. 110.

[22] S. L. Manzello and J. C. Yang, "An experimental study of a water droplet impinging on a liquid surface," Exp. Fluids, vol. 32, pp. 580589, 2002.

[23] S. P. Lin and R. D. Reitz, "Drop and spray formation from a liquid jet," Annu. Rev. Fluid Mech., vol. 30, pp. 85-105, 1998.

[24] P. J. A. Frinking and N. de Jong, "Acoustic modeling of shellencapsulated gas bubbles," Ultrasound Med. Biol., vol. 24, no. 4, pp. 523-533, 1998.

[25] M. Postema and G. Schmitz, "Ultrasonic fragmentation of microbubbles: a theoretical approach of the flash in flash-echo," Proc. IEEE Eng. Med. Biol. Soc., vol. accepted, 2005.

Michiel Postema e-mail: postema@ieee.org 

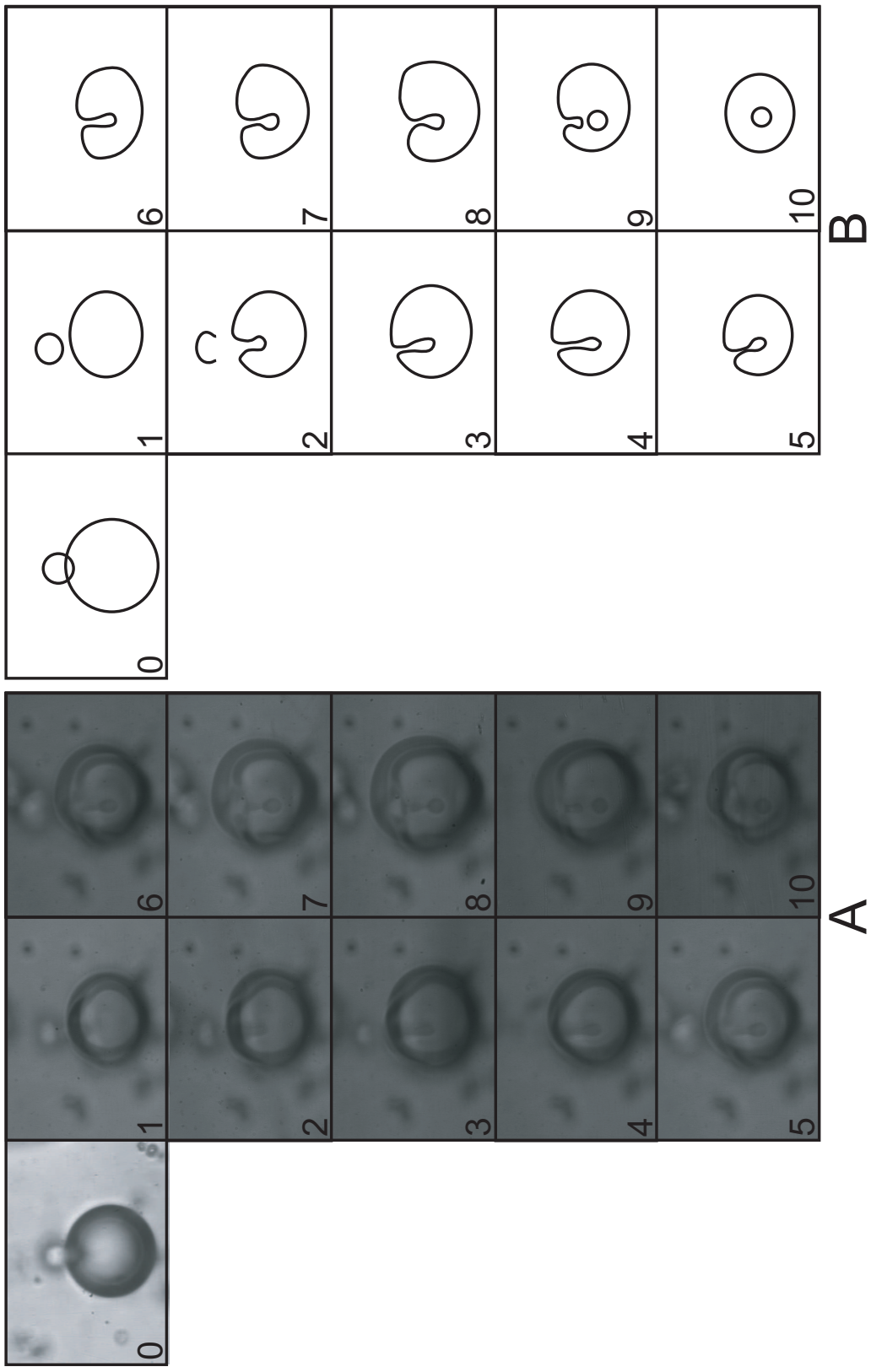

Fig. 3. Ultrasound-induced formation of an antibubble (A) and a schematic representation thereof (B). Each frame corresponds to a $71 \times 52 \mu \mathrm{m}$ area. Frame 0 was captured before ultrasound arrival. Frames 1-10 cover $5 \mu \mathrm{s}$, captured during insonification. This corresponds to four frames per ultrasonic cycle. The depth-of-field is approximately $5 \mu \mathrm{m}$, causing a stacked representation of three-dimensional microobjects. In frame 0 , a big free gas microbubble with an $30 \mu \mathrm{m}$ diameter is seen just below a $7.5 \mu \mathrm{m}$ Quantison ${ }^{\mathrm{TM}}$ microbubble that is slightly out of optical focus. After ultrasound arrival, presumably gas release takes place from the Quantison ${ }^{\mathrm{TM}}$ microbubble. This new microbubble is seen to expand and contract in the following frames. The two microbubbles interact, leading to an instability at the surface of the big free gas microbubble (frame 2). This instability has the form of a re-entrant jet protruding into the gas microbubble. The inward protrusion grows until frame 4 . Between frames 5 and 8 , the cylindrical protrusion drains. In frame 9 , a $5 \mu \mathrm{m}$ droplet is left inside the gas microbubble, while the protrusion retracts. Frame 10 shows the resulting antibubble: a spherical liquid core inside a spherical gas encapsulation. 